\title{
A Practical Approach to Software Continuous Delivery Focused on Application Lifecycle Management
}

\author{
Everton Gomede, Rafael Thiago da Silva and Rodolfo Miranda de Barros \\ Department of Computer Science \\ State University of Londrina \\ Londrina, Paraná, Brazil \\ e-mail: \{evertongomede, rafathiago\}@gmail.com, rodolfo@uel.br
}

\begin{abstract}
To deliver quality software continuously is a challenge for many organizations. It is due to factors such as configuration management, source code control, peer-review, delivery planning, audits, compliance, continuous integration, testing, deployments, dependency management, databases migration, creation and management of testing and production environments, traceability and data post-fact, integrated management, process standardization, among others. To overcome these challenges, this paper presents a continuous delivery process that promotes artefacts produced by developers, in a managed fashion, to production environment, allowing bidirectional traceability between requirements and executables, and integrating all activities of software development using the concepts of Application Lifecycle Management. As a result, we obtained an ecosystem of tools and techniques put into production in order to support this process 1 .
\end{abstract}

Keywords-Continuous Delivery; Process Quality; Application Lifecycle Management

\section{INTRODUCTION}

Software Delivery Process (SDP) consists of several tasks in order to promote artefacts created into the production environment (servers where an executable is installed to delivery features to the users) [1]. These ones can occur in either environment, producer or consumer. Due to the unique characteristics of each software product, a general process to various contexts probably cannot be set. Therefore, we should interpret a SDP as a framework to be customized according to the requirements and characteristics of each product (Software Delivery Process, in this context, is a part of Software Development Process).

This customization usually causes a manual execution of SDP [2]. Production environment is configured in a manual way by the infrastructure team using terminals and/or thirdparty tools. Artefacts are copied from a continuous integration server to a production environment and possibly some data and/or metadata are adjusted before software is released.

However, this process has some weaknesses. Predictability is the first one, because it increases risk, related with lost business transactions, and downtime whether any unexpected situation occurs [3]. Additionally, the repeatability factor may compromise the diagnosis of post-deployment problems [2]. Finally, this process is not auditable and it does not allow

${ }^{1}$ DOI reference number: 10.18293/SEKE2105-126 the recovery of information about all events that were held to deliver a version.

There is a growing interest in practices to overcome these problems [4]. Such practices are known as Software Continuous Delivery (SCD), defined as the ability to publish software whenever necessary. This publication may be weekly, daily or every change sent to the code repository. The frequency is not important, but the ability to deliver when it is necessary [2].

This approach has great importance in software development because it helps who is in charge of delivering to understand better their process and, consequently, improve it. Such improvements can be in terms of automation, decrease of the delivery time, rework and risk reduction, or others. Among them, the main is the ability to have a version of software, ready for delivery, each new code added to the repository.

Additionally, the management of the activities of application lifecycle, support this approach and help all team members to improve their process [2], [4]. Application Lifecycle Management (ALM) has been proposed with the objective to provide a comprehensive technical solution for monitoring, controlling and managing software development over the whole application lifecycle [5].

Thus, we propose a question: Are the concepts of ALM aiming at Software Continuous Delivery also an effective utility for improving the software process? We try to answer this question by describing the results and experiences from the introduction of a Continuous Software Delivery solution complemented by techniques of Application Lifecycle Management in a financial industry company.

In this context, we present a practical approach to address the problems of software continuous delivery and application lifecycle management. The main objective is to contribute with a setup of servers, process, techniques and tools that assist to deliver software continuously. In addition, some recommendations and further work are discussed. Issues related with software architecture, project management and other dimensions of software development were omitted.

Therefore, this article was divided into five sections, including this introduction. In Section II, we present fundamental concepts and related works. In Section III, we present an approach to Software Continuous Delivery focused on Application Lifecycle Management. In Section IV, we present the results. Finally, in Section V, we present conclusions, 
recommendations and suggestions for future work.

\section{FUndAMENTAL CONCEPTS AND RELATED WORKS}

There is a relation between quality of software products and quality of the process used to build them. Implementation of a process aims to reduce rework, delivery time and increase product quality, productivity, traceability, predictability and accuracy of estimates [2]. In general, a software development process contains the activities shown in Fig. 1.

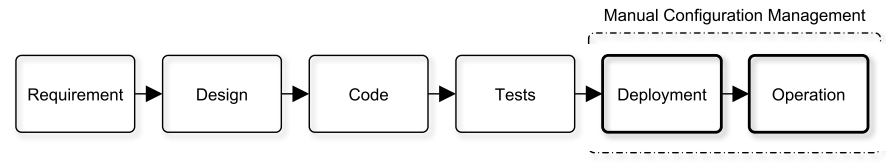

Figure 1. A simplified software development process [1], [2]

The configuration management tasks of deployment and operation activities, highlighted in Fig. 1, are usually performed manually [2]. This practice, according to Humble and Farley [2], is accompanied by anti-patterns:

- Deploying software manually: there should be only two tasks to perform manually; $(i)$ choose a version and (ii) choose the environment. These are goals to be achieved in a SCD [5].

- Deploying after development (requirement, design, code and tests) was complete: it is necessary to integrate all activities of the development process and put stakeholders working together since the beginning of the project.

- Manual configuration management of the production environments: all aspects of configured environments should be applied from a version control in an automated way.

In this context, some Software Continuous Delivery Practices arises. It is a developing discipline, which builds up software that can be released into production at any time, by minimizing lead-time [3].

To assist this type of software delivery approach, from construction to operation, Humble and Farley presents the Deployment Pipeline (DP), a standard to automate the process of SCD. Despite each organization may have an implementation of this standard, in general terms, it consists of the activities shown in Fig. 2.

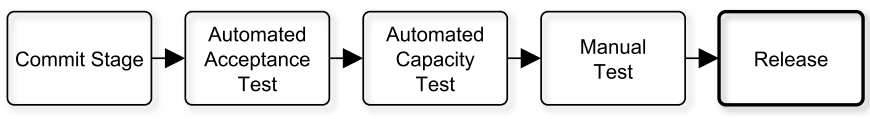

Figure 2. The deployment pipeline [2].

Over each change, artefacts are promoted to next instance of pipeline through a series of automated tasks. The first step of the pipeline is to create executables and installers from the code repository, in a process known as Continuous Integration (CI). Other activities perform a series of tests to ensure that the executable can be published. If the release candidate passes all tests and criteria, then it can be published [2].
To implement this pipeline, some approaches were presented. Among them, Krusche and Alperowitz [6] described the implementation of a SCD process to multiple projects. Their goal was to obtain the ability to publish software to their clients with just a few clicks. The main contribution of this work was to show that developers who have worked on projects with SCD, understood and applied the concepts, being convinced from the benefits of it.

Bellomo et al. [7] presented an architectural framework together with tactics to projects that address SCD. The main contribution of this work is a collection of SCD tactics in order to get software products performing with a higher level of reliability and monitoring into production environment.

Fitzgerald and Stol [4] published trends and challenges related to what the authors called "Continuous *", which is, all topics related to software delivery that can be classified as continuous. The authors addressed issues such as; Continuous Integration (CI), Continuous Publication (PC), Continuous Testing (CT), Continuous Compliance (CC), Continuous Security (SC), Continuous Delivery (EC), among others. An important point of this paper is the distinction between the Continuous Delivery and Continuous Publication. According to the authors, Continuous Publication is ability to put into production software products in an automated manner. This definition is complementary to the software continuous delivery definition given above.

Although all these works have a practical nature, none of them showed which tools were used, which recommendations to similar scenarios and which were the techniques used during deployment. Therefore, the work presented in this paper seeks to fill these gaps.

\section{A PRACTICAL APPROACH}

\section{A. Main Proposal}

The Fig. 3 shows all macro elements involved in approach. In the first line, there are two ones: (i) Development, representing the timeline of software development and (ii) Operation, representing the timeline of software operation. The pipeline of SCD is between both.

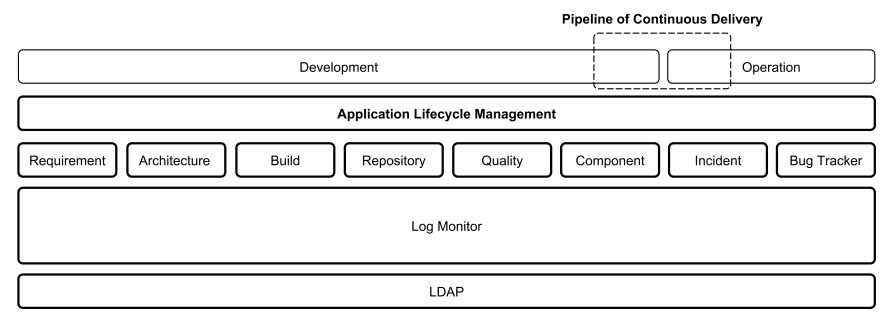

Figure 3. The big picture.

In the second and third line there are an ALM and activities of software development and operation. Requirement, architecture, build, repository, quality and component are related with development, meanwhile incident and bug tracker are related with operation. Log Monitor is used to collect and to integrate, in an automated way, information about all elements. LDAP is used to allow a single point of authentication and authorization between all components. 


\section{B. Infrastructure}

To provide an infrastructure that allows the Software Continuous Delivery is the main goal of setup shown in Fig. 4. It has 4 areas: (i) Commit Stage (CS), (ii) Quality Assurance (QA), (iii) Staging (ST) and (iv) Production (PD).

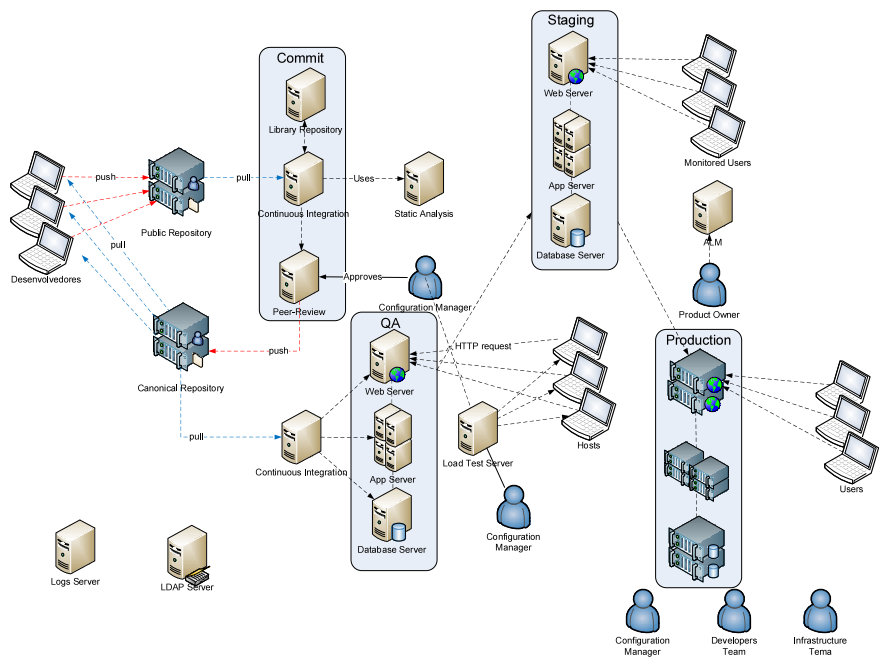

Figure 4. An overview of a setup of servers and areas.

\section{Areas}

The Commit Stage (CS) has primary responsibility to implement continuous integration of all code reviews sent to the repository. This area consists of the following services:

- $\quad$ Public Code Repository

- Purpose: to get code reviews that have not been approved.

- Tool: Git (git-scm.com).

- Technique: it has a single branch, called master, which receives revisions of all developers.

- Continuous Integration

- Purpose: to integrate all code reviews sent to the server.

- Tool: Jenkins (jenkins-ci.org) and Maven (maven.apache.org)

- Technique: it does integration performing unit testing and adding first acceptance step in peerreview server.

- Static Analysis

- Purpose: to make code analysis generating quality reports.

- Tool: SonarQube (sonarqube.org).

- Technique: each integration performs a series of tests, such as size metrics, complexity, test coverage, dependency calculation, among others. Creates a baseline quality of the project.

- Peer-Review

- Purpose: to enable promotion/rejection of codes from public to canonical repository.

- Tool: Gerrit (code.google.com/p/gerrit).

- Technique: approval of two steps, the first being carried out by continuous integration server and the second by the configuration manager. If the review through both sides, code is promoted to canonical repository.

- Canonical Repository

- Purpose: to receive approved code reviews.

- Tool: Git (git-scm.com).

- Technique: it has a single branch, called master, which receives revisions of peer-review server.

- Repository Libraries.

- Purpose: to store libraries and components used in integration.

- Tool: Nexus (sonatype.org/nexus).

- Technique: libraries and components are installed automatically or manually on the server being available for use at the time of integration.

The layout and operation of Commit Area are shown in Fig. 5 .

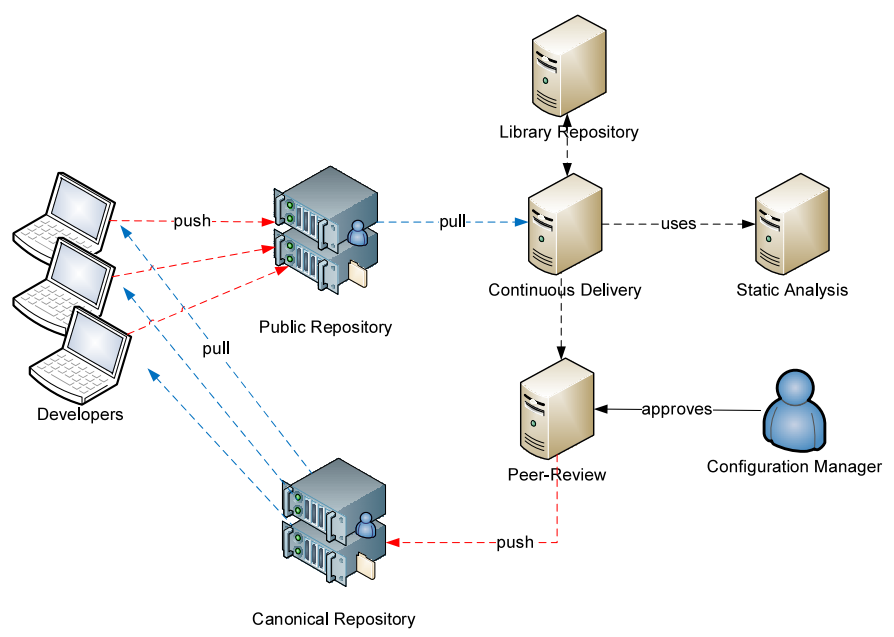

Figure 5. Commit Stage Area (CS).

The Quality Assurance Area (QA) has the main purpose of performing all automated tests and allow Quality Manager perform manual tests, such as exploratory testing [2]. This area consists of the following services:

- Continuous Integration

- Purpose: to obtain a copy of the code and perform integration, functional and automated load tests.

- Tool: Jenkins and Maven (maven.apache.org).

- Technique: get a copy of canonical repository to generate executable, install them into library server, application servers and database server. After that, execute integration, functional and load tests.

- $\quad$ Page Servers, Application and Database

- Purpose: to host application to test

- Tools: may vary according to the technology used; Wildfly (wildfly.org) and MSSQL are some examples.

- Technique: can vary depending on the technology used (to install and configure, basically). 
- Load Test

- Purpose: to perform a load test against the page servers, application and database.

○ Tool: Jmeter (jmeter.apache.org) and Vagrant (vagrantup.com).

- Technique: it performs script created by quality manager allocating hosts as required to test. It generates a supported load from baseline.

6.

The Operation of Quality Assurance area is shown in Fig.

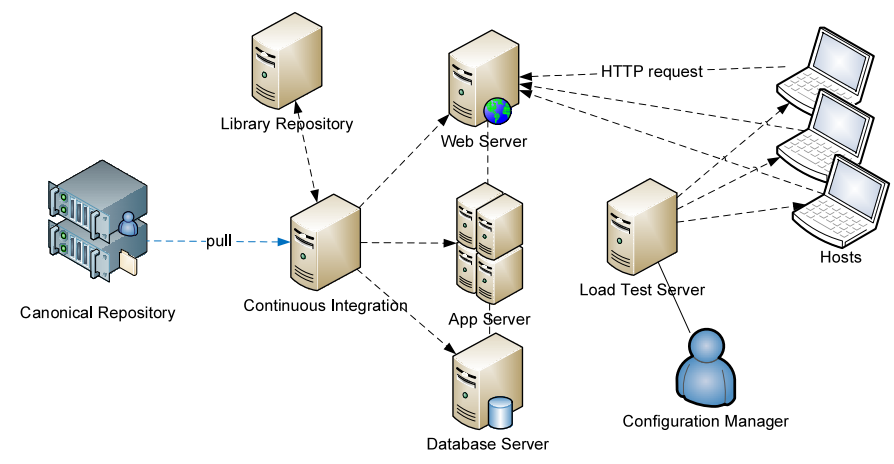

Figure 6. Quality Assurance Area (QA).

Staging Area aims to provide for monitoring users and product owners an environment as close as possible to production environment, so they perform approval tests. These ones are related to user experience and their perception regarding how software product meets specified requirements. This area has a copy of operating environments, both in terms of operating systems, tools and settings, and in terms of data. Monitored users are the ones chosen to perform approval tests in a monitoring way. Occasionally, they are in the product owner role. The Fig. 7 shows this area.

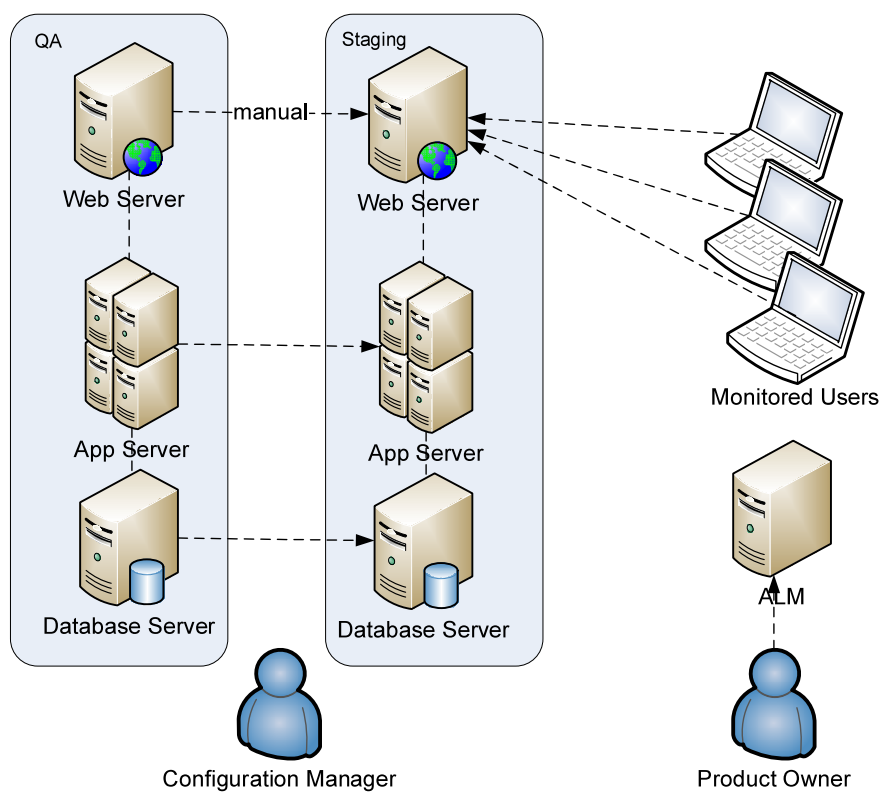

Figure 7. Staging Area (ST).
Finally, configuration manager makes promotion from Staging Area artefacts to Production Area manually by Configuration Manager. However, developers and infrastructure staff are present to perform this task. The Fig. 8 shows this area.

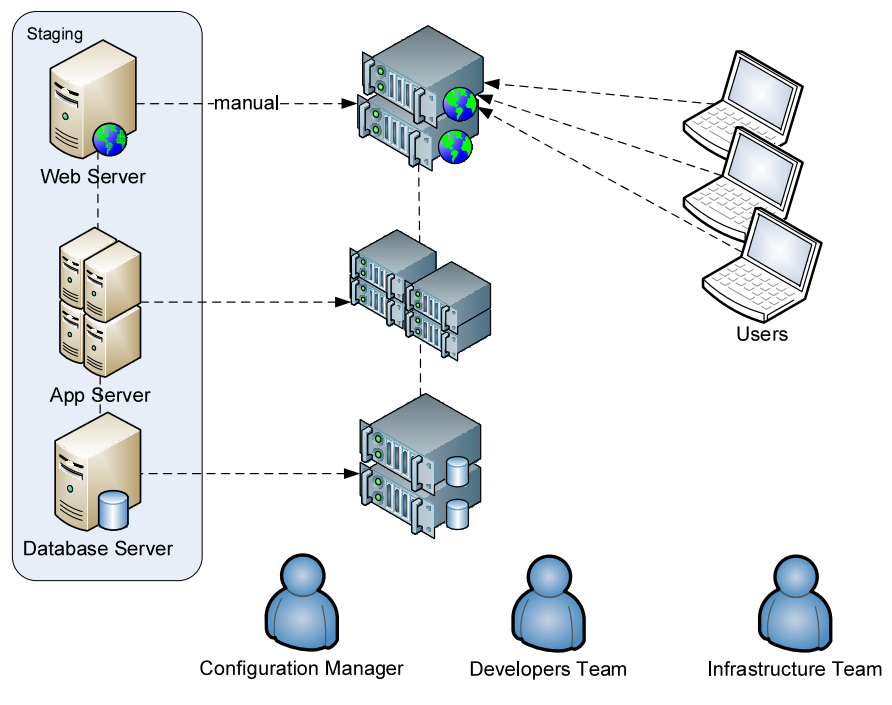

Figure 8. Production Area (PD).

Also, the following servers were used: (i) Log Server and (ii) LDAP Server. The first has a very important function in the setup; to get all events occurred by indexing logs. This assists the diagnosis, providing information to reporting, alerts and dashboard. The tool used in this case was Splunk. The second server has a function to allow authentication and authorization for all setup servers. This is necessary because it is costly to maintain users across all the servers involved in an individualized way, in addition this increase security flaws. The tool used in this case was OpenLDAP (openldap.org).

\section{Tools}

The Tab. I summarizes all tools used with its URL. These tools are used to Configuration Management (Git, Gerrit, Nexus, Flywaydb and Vagrant), Continuous Integration (Jenkins and Maven), Quality Assurance (SonarQube and Jmeter), Application Lifecycle Management (Redmine) and infrastructure (Splunk and OpenLDAP).

TABLE I. TOOLS USED.

\begin{tabular}{lll}
\hline Goal & Name & URL \\
\hline Continuous Integration & Jenkins & jenkins-ci.org \\
Source Repository & Git & git-scm.com \\
Build & Maven & maven.apache.org \\
Gathering Logs & Splunk & splunk.com \\
Peer-Review & Gerrit & code.google.com/p/gerrit \\
Static Analysis & SonarQube & sonarqube.org \\
Load Test & Jmeter & jmeter.apache.org \\
Library Repository & Nexus & sonatype.org/nexus \\
ALM & Redmine & redmine.org \\
Database Migration & Flywaydb & flywaydb.org \\
Automated Installation & Vagrant & vagrantup.com \\
Authentication/Authorization & OpenLDAP & openldap.org \\
Architecture & Enterprise Architect & sparxsystems.com.au \\
\hline
\end{tabular}

These tools were used because they are open/free software or a well known tool among developers. 


\section{RESUlts}

Some results about this approach are related to automation of many delivery tasks, coming out in a more predictable and managed process. Another aspect, related to collaboration, is due to communication between developers and infrastructure team was increased in all aspects of the process, since planning of a feature until its publication. These results are classified in a process maturity level [2], as shown in Fig. 9.

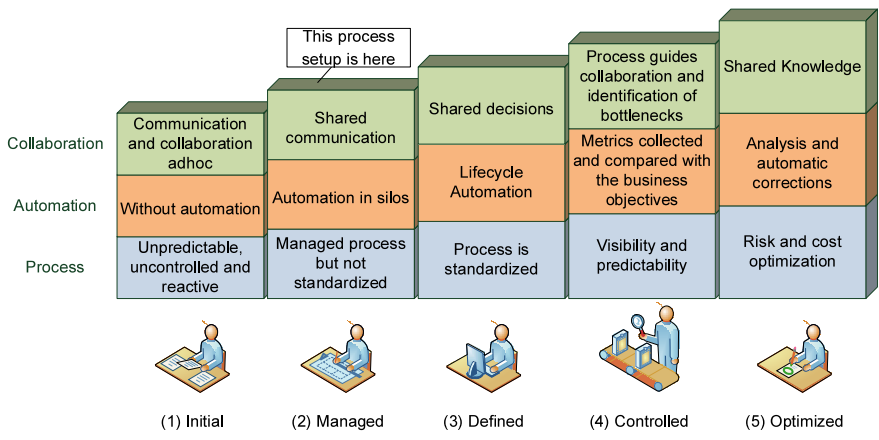

Figure 9. Process maturity level [2].

The Fig. 10 shows the peer-review authorization. It helps to protect the canonical repository and increase the quality of code for 3 reasons: (i) improve the quality of commits, each member tends to be more careful with a code that will be evaluate by another one, (ii) allow to put a sequence in commits avoiding collisions and wrong commits, (iii) allow to configuration manager to decide if the code can be promoted or not ${ }^{2}$.

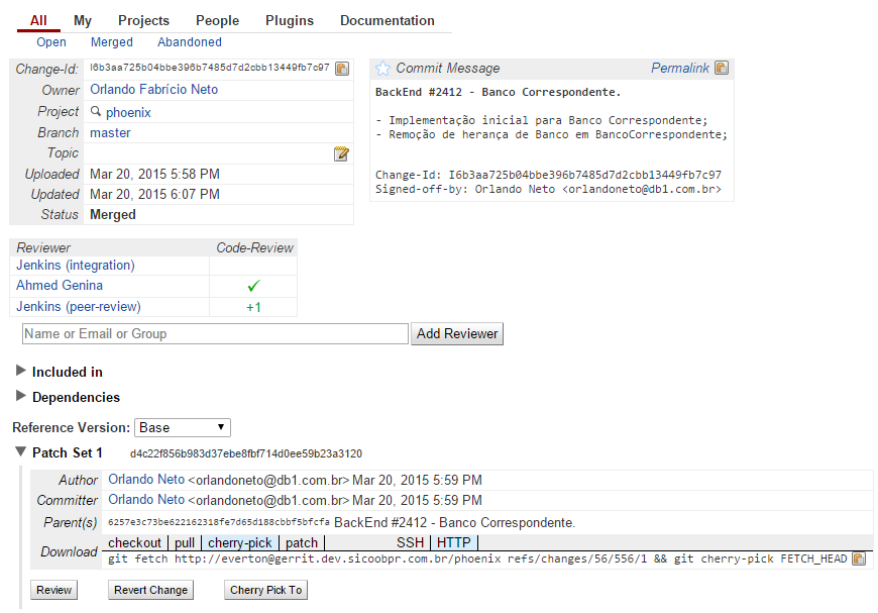

Figure 10. The peer-review authorization.

The Fig. 11 shows the integration of ALM tool (Redmine) with the canonical repository. This allow traceability between requirement, code, peer-review and repository. Among benefits, some of them are: $(i)$ vision end to end (requirement to executable), (ii) fast diagnosis in case of mistake during development (requirement, code, commit, release and other

\footnotetext{
${ }^{2}$ There are some words in portuguese because this picture was collected from a real situation in a brazilian company. The same situation occurs in the figures $10,12,13$ and 14
}

mistakes) and (iii) integrated management of application lifecycle (requirement, models, code, issues, peer-review, release, incidente, bugs, schedule, documents, wikis, forums, reports, components, library, repository).

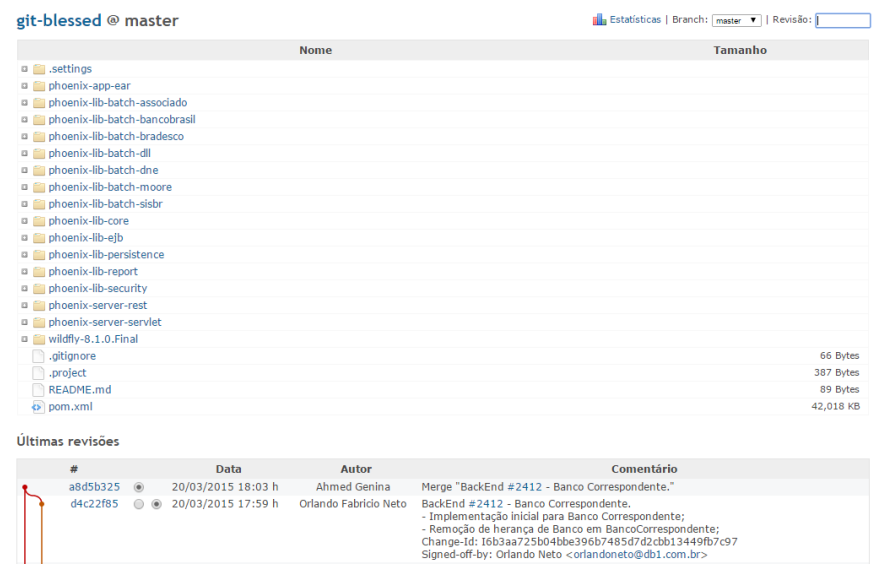

Figure 11. The integration of ALM tool with canonical repository.

The Fig. 12 shows a way, using some concepts of TOGAF 3 , to developer the requirements (ecosystem, vision, requirement, rules, use case). A fashion used to link an element of Enterprise Architect (EA) and the ALM tool was an ID, put in each one (GNF001, for instance).

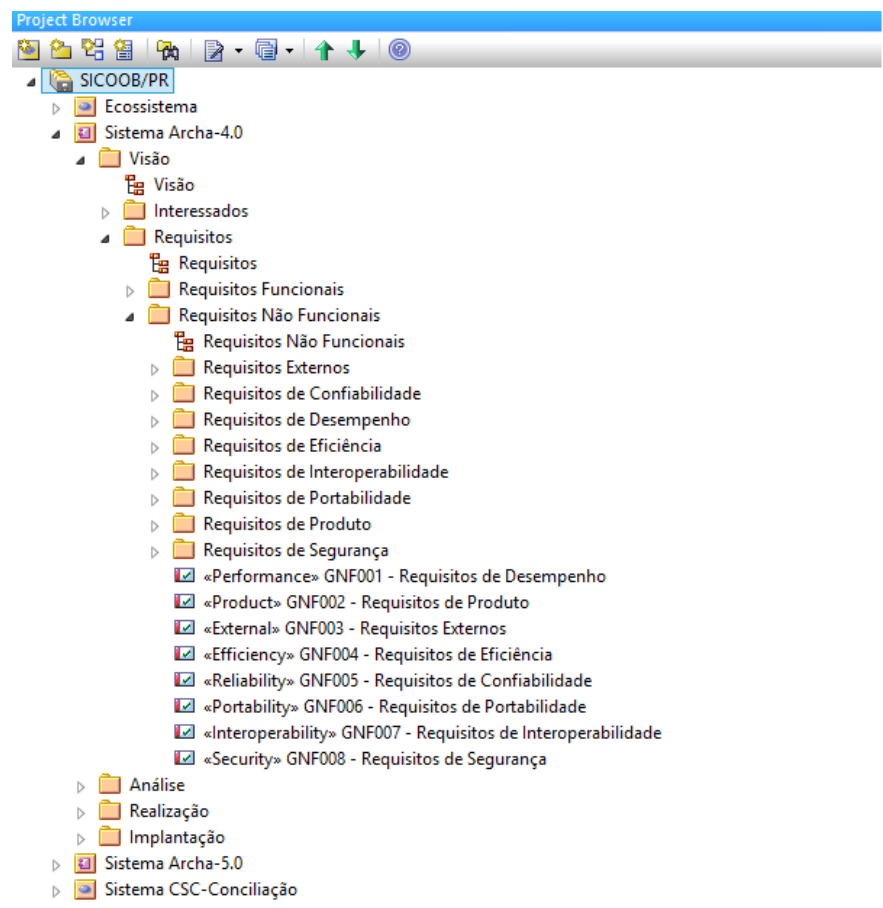

Figure 12. A way to developer requirements using some concepts of TOGAF.

The Fig. 13 shows the IC tool. Besides of all aspects of IC, there are two related with quality, specifically with

\footnotetext{
${ }^{3}$ The Open Group Architecture Framework (TOGAF) is a framework to enterprise architecture which provides an approach for designing, planning, implementing, and governing an enterprise information technology architecture. To see more, www.opengroup.org/togaf
} 
tests: $(i)$ snapshot of automated functional test, using Allure Framework ${ }^{4}$ and (ii) performance test, allowing identify the performance of application during the builds evolution. The first one is related with audit questions, as create evidence about functional tests and the second one with technical questions, as performance of some elements of application (queries, class, methods, algorithms).
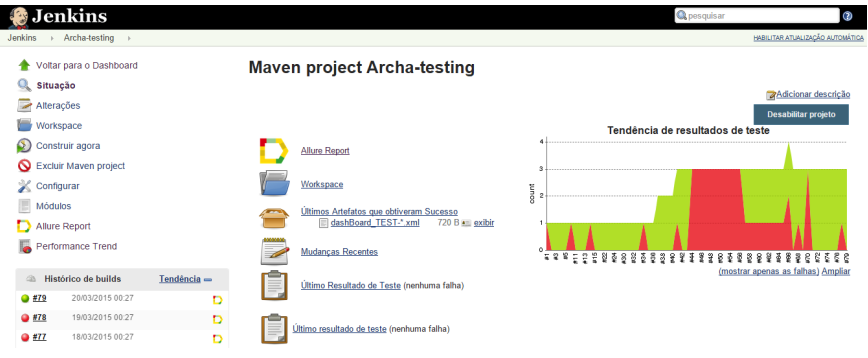

Figure 13. The IC tool.

The Fig. 14 shows the tool used to collect logs from all elements. The importance of this tool is related with diagnosis of all environments: $(i)$ development and (ii) operation. There are a lot of options of tools, for instance, Fluentd ${ }^{5}$ and Papertrail ${ }^{6}$. The important is not the tool, but the concept and to get the benefits of use. In case of doubt to choose one, we recommend a method called Analytic Hierarchy Process (AHP). More details about its use were shown in [8].

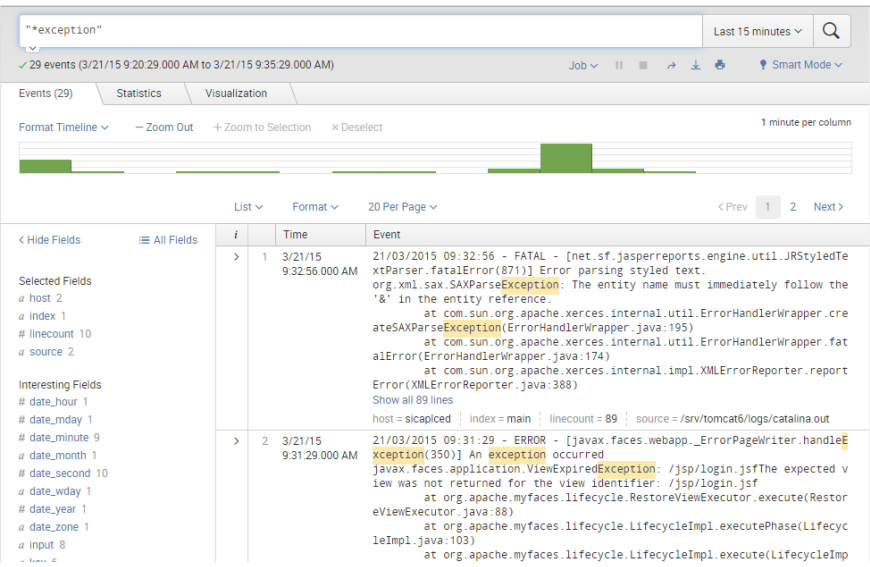

Figure 14. The tool used to collect logs from all elements.

In summary, the servers setup, tools and techniques were used to support the main objective that is put software in operation in a managed fashion, reducing risk, rework and increasing traceability, management, predictability and so forth.

As a macro result, the importance of this approach is related with:

- Traceability and data post-fact: to allow to link all elements using during software development process (requirement, models, code, versions) and generate a snapshot of all activities from each build (peer-review, tests, deployment).

\footnotetext{
${ }^{4}$ github.com/allure-framework/

5 fluentd.org

${ }^{6}$ papertrailapp.com
}

- Integrated management: all team members can use the information generated by ALM tool, like releases, issues, bugs, incidents, documents using only a local.

- Process standardization: a standard was created and it is useful to communicate and help team to understand better its process, allowing thus, a possibility of quality increase.

\section{CONCLUSION}

This work presented a practical approach that can be used in similar processes. Additionally, among the contributions can be mentioned (i) a set of tools evaluated and (ii) a set of techniques, that can be used for organizations that do not use this type of approach, as for those which already have a higher level of maturity.

Moreover, some further work may be developed to improve setup provided in this article. The first one aims to get a strategy for publication with less impact in terms of unavailability of software products, including deployment across different timezones. The second one is linked with multiple projects scenarios. We can analyze how the artefacts, from several projects, are promoted to production by the same team.

Finally, this article has a practical purpose. However, to implement continuous delivery and application lifecycle management involves more than installing some tools and automate some tasks. It depends on effective collaboration among all of those involved in the delivery process, senior management support and especially the desire of stakeholders in become the changes a reality.

\section{REFERENCES}

[1] M. V. Mantyla and J. Vanhanen, "Software Deployment Activities and Challenges - A Case Study of Four Software Product Companies," 2011 15th European Conference on Software Maintenance and Reengineering, Mar. 2011, pp. 131-140.

[2] J. Humble and F. David, Continuous Delivery: Reliable Software Releases through Build, Test, and Deployment Automation. ser. AddisonWesley Signature Series. Pearson Education, 2010.

[3] T. Ernawati and D. R. Nugroho, "IT Risk Management Framework Based on ISO 31000:2009," International Conference on System Engineering and Technology, vol. 11, 2012, pp. 1-8.

[4] B. Fitzgerald, "Continuous Software Engineering and Beyond : Trends and Challenges Categories and Subject Descriptors," RCoSE 14, 2014, pp. $1-9$.

[5] H. Lacheiner and R. Ramler, "Application Lifecycle Management as Infrastructure for Software Process Improvement and Evolution: Experience and Insights from Industry," 2011 37th EUROMICRO Conference on Software Engineering and Advanced Applications, Aug. 2011, pp. 286-293.

[6] S. Krusche and L. Alperowitz, "Introduction of Continuous Delivery in Multi-Customer Project Courses Categories and Subject Descriptors," ICSE Companion 14, 2014, pp. 335-343.

[7] S. Bellomo, N. Ernst, R. Nord, and R. Kazman, "Toward Design Decisions to Enable Deployability: Empirical Study of Three Projects Reaching for the Continuous Delivery Holy Grail," 2014 44th Annual IEEE/IFIP International Conference on Dependable Systems and Networks, Jun. 2014, pp. 702-707.

[8] E. Gomede and R. M. Barros, "A Non-Intrusive Process to Software Engineering Decision Support focused on increasing the Quality of Software Development," The 25th International Conference on Software Engineering and Knowledge Engineering, Boston, MA, USA, June 2729, 2013., Jun. 2013, pp. 95-100. 